TẠP CHÍ KHOA HỌC ĐẠI HỌC TÂN TRÀO

ISSN: $2354-1431$

http://tckh.daihoctantrao.edu.vn/

\title{
A NEW EXTENSION OF PARAMETER CONTINUATION METHOD FOR SOLVING OPERATOR EQUATIONS OF THE SECOND KIND
}

\author{
Ngo Thanh Binh ${ }^{1, *}$ \\ ${ }^{1}$ Nam Dinh University of Technology Education, Vietnam \\ *Email address: ntbinhspktnd@gmail.com \\ https://doi.org/10.51453/2354-1431/2021/521
}

Article info

Recieved:

27/3/2021

Accepted:

$3 / 5 / 2021$

\section{Keywords:}

Parameter continuation method, Monotone operator, Contractive operator, Operator equations of the second kind, Approximate solution.

\begin{abstract}
:
In this paper, we propose an extension of the parameter continuation method for solving operator equations of the second kind. By splitting of the operator into a sum of two operators: one monotone, Lipschitz-continuous and one contractive, the applicability of the method is broader. The suitability of the proposed approach is presented through an example.
\end{abstract}




\title{
TẠP CHÍ KHOA HỌC ĐẠI HỌC TÂN TRÀO
}

ISSN: $2354-1431$

http://tckh.daihoctantrao.edu.vn/

\section{MộT SỰ MỞ RộNG MỚI CỦA PHƯƠNG PHÁP THÁC TRIỂN THEO THAM SỐ GIẢI PHƯƠNG TRÌNH TOÁN TỦ LOẠI HAI}

\author{
Ngô Thanh Bình ${ }^{l, *}$ \\ ${ }^{1}$ Truoòng Đại học Su phạm Kỹ thuật Nam Định, Việt Nam \\ *Địa chi email: ntbinhspktnd@gmail.com \\ https://doi.org/ 10.51453/2354-1431/2021/521
}

\section{Thông tin bài viết}

Ngày nhận bài:

$27 / 3 / 2021$

Ngày duyệt đăng:

$3 / 5 / 2021$

Tù̀ khóa: Phuoong pháp thác triển theo tham số, Toán tủ đơn điệu, Toán tủ co, Phuoong trình toán tủ loại hai, Giải xấp xi.

\section{Tóm tắt}

Trong bài báo này, chúng tôi đề xuất một mở rộng mới của phương pháp thác triển theo tham số giải phương trình toán tử loại hai. Bằng cách tách toán tử thành tổng của hai toán tử: toán tử đơn điệu, liên tục Lipschitz và toán tử co, khả năng áp dụng của phương pháp được mở rộng. Sự phù hợp của cách tiếp cận đề xuất được trình bày thông qua một ví dụ.

\section{Introductions}

Parameter continuation method (PCM) was suggested and developed by Bernstein [1] and Schauder [3] which is the inclusion of the equation $P(x)=0$ into the one-parametric family of equations $G(x, \varepsilon)=0, \varepsilon \in[0,1]$ connecting the given equation $(\varepsilon=1)$ with a solvable equation ( $\varepsilon=0)$ and study the dependence of the solution from parameter. The PCM is a powerful technique for solving operator equations, see for example [57]. Gaponenko [2] introduced the PCM for solving operator equations of the second kind

$$
x+A(x)=f,
$$

where $A$ is a Lipschitz-continuous and monotone operator, which operates in an arbitrary Banach space $X$. The monotone operator in Banach space is defined as follows.

Definition 1.1. [2, Definition 2] The mapping A, which operates in the Banach space $X$ is called monotone if for any elements $x_{1}, x_{2} \in X$ and any $\varepsilon>0$ the following inequality holds

$$
\left\|x_{1}-x_{2}+\varepsilon\left[A\left(x_{1}\right)-A\left(x_{2}\right)\right]\right\| \geq\left\|x_{1}-x_{2}\right\| .
$$

Remark 1.1. [2, Remark 1] If $X$ is Hilbert space then the condition of monotonicity (2) is equivalent to the classical condition

$$
\left\langle A\left(x_{1}\right)-A\left(x_{2}\right), x_{1}-x_{2}\right\rangle \geq 0, \forall x_{1}, x_{2} \in X,
$$


where $\langle\cdot, \cdot\rangle$ is an inner product in the Hilbert space $X$.

We obtain the following result from the definition above.

Lemma 1.1. [2, Lemma] Assume that $A$ is a monotone mapping which operates in the Banach space $X$. Then for any elements $x_{1}, x_{2} \in X$ and any positive numbers $\varepsilon_{1}, \varepsilon_{2}, 0<\varepsilon_{1} \leq \varepsilon_{2} \leq 1$, the following inequality holds

$\left\|x_{1}-x_{2}+\varepsilon_{1}\left[A\left(x_{1}\right)-A\left(x_{2}\right)\right]\right\| \leq\left\|x_{1}-x_{2}+\varepsilon_{2}\left[A\left(x_{1}\right)-A\left(x_{2}\right)\right]\right\|$.

The results obtained by Gaponenko are summarized in Theorem 1 and Theorem 2.

Theorem 1.1. [2, Theorem 1] Suppose that the mapping A, which operates in the Banach space

$X$ is Lipschitz - continuous and monotone. Then the equation (1) has a unique solution for any

element $f \in X$.

The following iteration process is constructed to find approximate solutions of the equation (1).

$x_{i+1}=\underbrace{-\frac{1}{N} A\left(x_{i}\right)-\frac{1}{N} A\left(x_{j}\right)-\cdots-\frac{1}{N} A\left(x_{p}\right)}_{N \text { terms }}+f, i, j, \ldots, p=0,1, \ldots$.

The symbolic notation (3) should be understood as the following iteration processes, which consist of $N$ iteration processes

$$
\begin{gathered}
x_{i+1}=-\varepsilon_{0} A\left(x_{i}\right)+x_{j}^{(1)}, i=0,1,2, \ldots, \\
x_{j+1}^{(1)}=-\varepsilon_{0} A G_{1}^{-1}\left(x_{j}^{(1)}\right)+x_{l}^{(2)}, j=0,1,2, \ldots, \\
\cdots, \\
x_{p+1}^{(N-1)}=-\varepsilon_{0} A G_{1}^{-1} \cdots G_{N-1}^{-1}\left(x_{p}^{(N-1)}\right)+f, p=0,1,2, \ldots
\end{gathered}
$$

For simplicity, assume that $A(0)=0$ and the number of steps in each iteration scheme of the iteration process (3) is the same and equals $n$. Denoting $x(n, N) \equiv x_{n}$ as the approximate solutions of the equation (1), which is constructed by the iteration process (3). In this case, Gaponenko received the error estimations of approximate solutions of the equation (1), which are presented in the following theorem.

Theorem 1.2. [2, Theorem 2] Assume that the conditions of Theorem 1.1 are satisfied. Then the sequence of approximate solutions $\{x(n, N)\}, n=1,2, \ldots \quad$ constructed by iteration process (3) converges to the exact solution $x$ of the equation (1). Moreover, the following estimates hold

$$
\|x(n, N)-x\| \leq \frac{q^{n+1}}{1-q} \frac{e^{q N}-1}{e^{q}-1}\|f\| .
$$

where $L$ is Lipschitz coefficient of the operator $A, N$ is the smallest natural number such that $q=\frac{L}{N}<1, n=1,2, \ldots$.

\section{MAIN RESULTS}

Consider the general operator equation (1)

$$
x+A(x)=f,
$$

where $A$ is a nonlinear operator from a Banach space $X$ into $X, f$ is a given function in $X$. Without loss of generality, one can express the operator $A$ as a composition of two operators $A_{1}$ and $A_{2}$. Then the equation (1) can be rewritten as follow

$$
x+A_{1}(x)+A_{2}(x)=f .
$$

Theorem 2.1. Assume that $A_{1}$ is a Lipschitzcontinuous and monotone operator, $A_{2}$ is a contractive operator. Then the equation (1) has a unique solution.

Proof. We take a minimal natural number $N$ such that $q_{1}=\varepsilon_{0} L<1, \varepsilon_{0}=\frac{1}{N}$, where $L$ is the Lipschitz coefficient of the operator $A_{1}$. The equation (6) can be written as the following form

$$
x+N \varepsilon_{0} A_{1}(x)+A_{2}(x)=f .
$$

Consider the following subsidiary problems.

Problem $1 \quad(N=1)$. Consider the operator equation

$$
x+\varepsilon_{0} A_{1}(x)+A_{2}(x)=f .
$$

We shall carry out a change of variable

$$
x^{(1)}=x+\varepsilon_{0} A_{1}(x) \equiv G_{1}(x) .
$$

We have 


$$
\begin{aligned}
\left\|\varepsilon_{0} A_{1}(x)-\varepsilon_{0} A_{1}(\bar{x})\right\| & \leq \varepsilon_{0} L\|x-\bar{x}\| \\
& =q_{1}\|x-\bar{x}\|, \forall x, \bar{x} \in X .
\end{aligned}
$$

Hence $\varepsilon_{0} A_{1}$ is a contractive operator with contraction coefficient equal to $q_{1}=\varepsilon_{0} L<1$. Then the equation (9) has a unique solution for any $x^{(1)} \in X$, i.e., the operator $G_{1}^{-1}\left(x^{(1)}\right)$ is determined in the whole space $X$. By virtue of the monotonicity of the operator $A_{1}$, the operator $G_{1}^{-1}$ is Lipschitz - continuous with Lipschitz coefficient equal to 1 . Indeed, for any $x^{(1)}, \bar{x}^{(1)} \in X$, we have

$$
\begin{aligned}
\left\|G_{1}^{-1}\left(x^{(1)}\right)-G_{1}^{-1}\left(\bar{x}^{(1)}\right)\right\| & =\|x-\bar{x}\| \\
& \leq\left\|x-\bar{x}+\varepsilon_{0}\left[A_{1}(x)-A_{1}(\bar{x})\right]\right\| \\
& =\left\|x^{(1)}-\bar{x}^{(1)}\right\| .
\end{aligned}
$$

After changing the variable (9), the equation (8) will take the following form

$$
P_{1}\left(x^{(1)}\right) \equiv x^{(1)}+A_{2} G_{1}^{-1}\left(x^{(1)}\right)=f .
$$

For any $x^{(1)}, \bar{x}^{(1)} \in X$, we have

$$
\begin{aligned}
& \left\|A_{2} G_{1}^{-1}\left(x^{(1)}\right)-A_{2} G_{1}^{-1}\left(\bar{x}^{(1)}\right)\right\| \\
& \leq q_{2}\left\|G_{1}^{-1}\left(x^{(1)}\right)-G_{1}^{-1}\left(\bar{x}^{(1)}\right)\right\| \leq q_{2}\left\|x^{(1)}-\bar{x}^{(1)}\right\|,
\end{aligned}
$$

where $q_{2}$ is contraction coefficient of the operator $A_{2}$. Thus $A_{2} G_{1}^{-1}$ is a contractive operator with contraction coefficient equal to $q_{2}<1$. Then the equation (10) has a unique solution for any $f \in X$. Consequently, the equation (8) has a unique solution $x\left(\varepsilon_{0}\right)$ for any $f \in X$.

Problem $2(N=2)$. Consider the operator equation

$$
x+2 \varepsilon_{0} A_{1}(x)+A_{2}(x)=f .
$$

We shall carry out two changes of variables

$$
\begin{aligned}
& x^{(1)}=x+\varepsilon_{0} A_{1}(x) \equiv G_{1}(x), \\
& x^{(2)}=x^{(1)}+\varepsilon_{0} A_{1} G_{1}^{-1}\left(x^{(1)}\right) \equiv G_{2}\left(x^{(1)}\right) .
\end{aligned}
$$

For any $x^{(1)}, \bar{x}^{(1)} \in X$, we have

$$
\begin{aligned}
\left\|\varepsilon_{0} A_{1} G_{1}^{-1}\left(x^{(1)}\right)-\varepsilon_{0} A_{1} G_{1}^{-1}\left(\bar{x}^{(1)}\right)\right\| & \leq \varepsilon_{0} L\left\|x^{(1)}-\bar{x}^{(1)}\right\| \\
& =q_{1}\left\|x^{(1)}-\bar{x}^{(1)}\right\| .
\end{aligned}
$$

Hence $\varepsilon_{0} A_{1} G_{1}^{-1}$ is a contractive operator with contraction coefficient equal to $q_{1}<1$. Then the equation (12b) has a unique solution for any $x^{(2)} \in X$, i.e., the operator $G_{2}^{-1}$ is determined in the whole space $X$. By Lemma 1.1, for any $x^{(2)}, \bar{x}^{(2)} \in X$, we have

$$
\begin{aligned}
& \left\|G_{2}^{-1}\left(x^{(2)}\right)-G_{2}^{-1}\left(\bar{x}^{(2)}\right)\right\|=\left\|x^{(1)}-\bar{x}^{(1)}\right\| \\
& =\left\|x-\bar{x}+\varepsilon_{0}\left[A_{1}(x)-A_{1}(\bar{x})\right]\right\| \leq\left\|x-\bar{x}+2 \varepsilon_{0}\left[A_{1}(x)-A_{1}(\bar{x})\right]\right\| \\
& =\left\|x^{(1)}-\bar{x}^{(1)}+\varepsilon_{0}\left[A_{1} G_{1}^{-1}\left(x^{(1)}\right)-A_{1} G_{1}^{-1}\left(\bar{x}^{(1)}\right)\right]\right\|=\left\|x^{(2)}-\bar{x}^{(2)}\right\| .
\end{aligned}
$$

Thus the operator $G_{2}^{-1}$ is Lipschitz - continuous with Lipschitz coefficient equal to 1. After changing the variables (12a) and (12b), the equation (11) will take the following form

$$
P_{2}\left(x^{(2)}\right) \equiv x^{(2)}+A_{2} G_{1}^{-1} G_{2}^{-1}\left(x^{(2)}\right)=f .
$$

For any $x^{(2)}, \bar{x}^{(2)} \in X$, we have

$$
\left\|A_{2} G_{1}^{-1} G_{2}^{-1}\left(x^{(2)}\right)-A_{2} G_{1}^{-1} G_{2}^{-1}\left(\bar{x}^{(2)}\right)\right\| \leq q_{2}\left\|x^{(2)}-\bar{x}^{(2)}\right\| .
$$

Thus $A_{2} G_{1}^{-1} G_{2}^{-1}$ is a contractive operator with contraction coefficient equal to $q_{2}<1$. Then the equation (13) has a unique solution for any $f \in X$. Therefore the equation (11) has a unique solution $x\left(2 \varepsilon_{0}\right)$ for any $f \in X$.

Problem $N \quad(N>2)$. Consider the operator equation

$x+N \varepsilon_{0} A_{1}(x)+A_{2}(x) \equiv x+A_{1}(x)+A_{2}(x)=f$.

We shall carry out $N$ changes of variables

$x^{(1)}=x+\varepsilon_{0} A_{1}(x) \equiv G_{1}(x)$,

$x^{(2)}=x^{(1)}+\varepsilon_{0} A_{1} G_{1}^{-1}\left(x^{(1)}\right) \equiv G_{2}\left(x^{(1)}\right)$,

....

$x^{(N)}=x^{(N-1)}+\varepsilon_{0} A_{1} G_{1}^{-1} \cdots G_{N-1}^{-1}\left(x^{(N-1)}\right) \equiv G_{N}\left(x^{(N-1)}\right)$.

Similarily, we show that the operators $G_{3}^{-1}, G_{4}^{-1}, \ldots, G_{N}^{-1}$ are determined in the whole space $X$ and are Lipschitz - continuous with Lipschitz coefficients equal to 1 . Hence after the change of variables (15a)-(15d) the equation (14) will take the following form 
$P_{N}\left(x^{(N)}\right) \equiv x^{(N)}+A_{2} G_{1}^{-1} \cdots G_{N}^{-1}\left(x^{(N)}\right)=f$.

For any $x^{(N)}, \bar{x}^{(N)} \in X$, we have

$$
\begin{aligned}
& \left\|A_{2} G_{1}^{-1} \cdots G_{N}^{-1}\left(x^{(N)}\right)-A_{2} G_{1}^{-1} \cdots G_{N}^{-1}\left(\bar{x}^{(N)}\right)\right\| \\
& \leq q_{2}\left\|x^{(N)}-\bar{x}^{(N)}\right\| .
\end{aligned}
$$

Thus $A_{2} G_{1}^{-1} \cdots G_{N}^{-1}$ is a contractive operator with contraction coefficient equal to $q_{2}<1$. Then the integral equation (16) has a unique solution for any $f \in X$. Consequently, the equation (14) has a unique solution $x\left(N \varepsilon_{0}\right) \equiv x \in X$ for any $f \in X$, i.e. the equation (1) has a unique solution $x \in X$ for any $f \in X$.This completes the proof.

We now construct the iterative algorithm to find approximate solution of the operator equation (1). Firstly, we construct the iterative algorithm to find approximate solution of the Problem 1. The approximate solutions of the equation (10) are obtained by using the standard iteration process

$$
x_{j+1}^{(1)}=-A_{2} G_{1}^{-1}\left(x_{j}^{(1)}\right)+f, j=0,1,2, \ldots, x_{0}^{(1)}=f .
$$

At the same time at each step of above iteration process when calculating the value $G_{1}^{-1}\left(x_{j}^{(1)}\right)$ we will again use the standard iteration process

$$
x_{i+1}=-\varepsilon_{0} A_{1}\left(x_{i}\right)+x_{j}^{(1)}, i=0,1,2, \ldots, x_{0}=x_{j}^{(1)} .
$$

As a result, the approximate solutions of the equation (8) can be found by the following iteration processes

$$
\begin{aligned}
& x_{i+1}=-\varepsilon_{0} A_{1}\left(x_{i}\right)+x_{j}^{(1)}, i=0,1,2, \ldots, \\
& x_{j+1}^{(1)}=-A_{2} G_{1}^{-1}\left(x_{j}^{(1)}\right)+f, j=0,1,2, \ldots, x_{0}^{(1)}=f .
\end{aligned}
$$

Next, we construct the iterative algorithm to find approximate solution of the Problem 2. The approximate solutions of the integral equation (13) are obtained by using the standard iteration process

$$
x_{l+1}^{(2)}=-A_{2} G_{1}^{-1} G_{2}^{-1}\left(x_{l}^{(2)}\right)+f, l=0,1,2, \ldots, x_{0}^{(2)}=f .
$$

At the same time we will use "subsidiary" iteration processes to invert the operators $G_{1}, G_{2}$ at each step of this iteration process when calculating the value of $G_{1}^{-1} G_{2}^{-1}\left(x_{l}^{(2)}\right)$. Hence the approximate solutions of the integral equation (11) can be found by

iteration processes

$$
\begin{aligned}
& x_{i+1}=-\varepsilon_{0} A_{1}\left(x_{i}\right)+x_{j}^{(1)}, i=0,1,2, \ldots, \\
& x_{j+1}^{(1)}=-\varepsilon_{0} A_{1} G_{1}^{-1}\left(x_{j}^{(1)}\right)+x_{l}^{(2)}, j=0,1,2, \ldots, \\
& x_{l+1}^{(2)}=-A_{2} G_{1}^{-1} G_{2}^{-1}\left(x_{l}^{(2)}\right)+f, l=0,1,2, \ldots, x_{0}^{(2)}=f .
\end{aligned}
$$

Finally, we construct the iterative algorithm to find approximate solution of the Problem $N$. The approximate solutions of the integral equation (16) are obtained by using the standard iteration process $x_{p+1}^{(N)}=-A_{2} G_{1}^{-1} \cdots G_{N}^{-1}\left(x_{p}^{(N)}\right)+f, p=0,1,2, \ldots, x_{0}^{(N)}=f$.

At the same time we will use "subsidiary" iteration processes to invert the operators $G_{1}, G_{2}, \ldots, G_{N}$ at each step of this iteration process when calculating the value of $G_{1}^{-1} G_{2}^{-1} \cdots G_{N}^{-1}\left(x_{p}^{(N)}\right)$. Hence the approximate solutions of the equation (14) can be found by iteration processes

$$
\begin{aligned}
x_{i+1} & =-\varepsilon_{0} A_{1}\left(x_{i}\right)+x_{j}^{(1)}, i=0,1,2, \ldots, \\
x_{j+1}^{(1)} & =-\varepsilon_{0} A_{1} G_{1}^{-1}\left(x_{j}^{(1)}\right)+x_{l}^{(2)}, j=0,1,2, \ldots, \\
& \ldots, \\
x_{p+1}^{(N)} & =-A_{2} G_{1}^{-1} \cdots G_{N}^{-1}\left(x_{p}^{(N)}\right)+f, p=0,1,2, \ldots, x_{0}^{(N)}=f .
\end{aligned}
$$

The iteration processes (19a)-(19d) can be written as the following symbolic notation

$$
\begin{aligned}
x_{i+1}= & \underbrace{-\frac{1}{N} A_{1}\left(x_{i}\right)-\frac{1}{N} A_{1}\left(x_{j}\right)-\cdots-\frac{1}{N} A_{1}\left(x_{h}\right)}_{N \text { terms }}-A_{2}\left(x_{p}\right)+f, \\
& i, j, \ldots, p=0,1, \ldots .
\end{aligned}
$$

Assume that the number of steps in each iteration scheme of iteration processes (19a)-(19d) is the same and equals $n$. Let $x_{n}$ be approximate solutions of the equation (1). Note that $x_{n}$ depends on $N$. Hence we denote $x(n, N) \equiv x_{n}$. We have the following theorem.

Theorem 2.2. Let the assumptions of Theorem 2.1 be satisfied. Then the sequence of approximate solutions $\{x(n, N)\}, n=1,2, \ldots \quad$ constructed by iteration processes (19a)-(19d) converges to the exact solution $x \in X$ of the operator equation (1). 
Moreover, the following estimates hold $\|x(n, N)-x\| \leq \frac{1}{1-q_{2}}\left[\frac{q_{1}^{n+1}}{1-q_{1}} \frac{1-q_{2}{ }^{n+1}}{1-q_{2}} \frac{e^{q_{1} N}-1}{e^{q_{1}}-1}+q_{2}^{n+1}\right]\|f\|$,

where $N$ is the smallest natural number such that $q_{1}=\frac{L}{N}<1, \quad L \quad$ is Lipschitz coefficient of the operator $A_{1}, q_{2}$ is a contraction coefficient of the operator $A_{2}, n=1,2, \ldots$.

Proof. Without loss of generality, we assume that $A_{1}(0)=0, A_{2}(0)=0$. Let us consider successive problems $1,2, \ldots, N$. The approximate solutions of Problem 1 assumes are obtained by iteration processes (17a)-(17b). The values $G_{1}^{-1}\left(x_{j}^{(1)}\right)$ are calculated by using the iteration process (17a) with the error

$$
\left\|x_{n}-x^{*}\right\| \leq \frac{q_{1}^{n+1}}{1-q_{1}}\left\|x_{j}^{(1)}\right\|
$$

For any $k \in\{1,2, \ldots, n\}$, we have

$$
\begin{aligned}
\left\|x_{k}^{(1)}-x_{k-1}^{(1)}\right\| & =\left\|A_{2} G_{1}^{-1}\left(x_{k-1}^{(1)}\right)-A_{2} G_{1}^{-1}\left(x_{k-2}^{(1)}\right)\right\| \\
& \leq q_{2}\left\|x_{k-1}^{(1)}-x_{k-2}^{(1)}\right\| \leq \cdots \leq q_{2}^{k-1}\left\|x_{1}^{(1)}-x_{0}^{(1)}\right\|,
\end{aligned}
$$

so that

$$
\begin{aligned}
& \left\|x_{j}^{(1)}\right\| \leq\left\|x_{j}^{(1)}-x_{j-1}^{(1)}\right\|+\cdots+\left\|x_{1}^{(1)}-x_{0}^{(1)}\right\|+\left\|x_{0}^{(1)}\right\| \\
& \leq\left(q_{2}^{j-1}+q_{2}^{j-2}+\cdots+q_{2}+1\right)\left\|x_{1}^{(1)}-x_{0}^{(1)}\right\|+\left\|x_{0}^{(1)}\right\| \\
& \leq \frac{1-q_{2}^{j}}{1-q_{2}}\left\|x_{1}^{(1)}-x_{0}^{(1)}\right\|+\left\|x_{0}^{(1)}\right\| .
\end{aligned}
$$

Since $A_{1}(0)=0$, we have $G_{1}(0)=0+\varepsilon_{0} A_{1}(0)=0$

. Hence

$$
\begin{aligned}
\left\|x_{1}^{(1)}-x_{0}^{(1)}\right\| & =\left\|A_{2} G_{1}^{-1}\left(x_{0}^{(1)}\right)+f-x_{0}^{(1)}\right\| \\
& =\left\|A_{2} G_{1}^{-1}(f)-A_{2} G_{1}^{-1}(0)\right\| \leq q_{2}\|f\| .
\end{aligned}
$$

Then from above inequality it follows that

$$
\begin{aligned}
\left\|x_{j}^{(1)}\right\| & \leq \frac{1-q_{2}^{j}}{1-q_{2}}\left\|x_{1}^{(1)}-x_{0}^{(1)}\right\|+\left\|x_{0}^{(1)}\right\| \leq q_{2} \frac{1-q_{2}^{j}}{1-q_{2}}\|f\|+\|f\| \\
& \leq q_{2} \frac{1-q_{2}^{n}}{1-q_{2}}\|f\|+\|f\|=\frac{1-q_{2}^{n+1}}{1-q_{2}}\|f\| .
\end{aligned}
$$

Consequently, the values $G_{1}^{-1}\left(x_{j}^{(1)}\right)$ are calculated with the error $\Delta_{1}(n) \equiv \delta_{1}(n) \equiv\left\|x_{n}-x^{*}\right\| \leq \mu(n)$, where

$$
\mu(n)=\frac{q_{1}^{n+1}}{1-q_{1}} \frac{1-q_{2}^{n+1}}{1-q_{2}}\|f\| .
$$

Since $A_{2}$ is a contractive operator with contraction coefficient equal to $q_{2}<1$, the error $\Delta_{1}(n)$ in specifying the argument of the operator $A_{2}$ is equivalent to the error $q_{2} \Delta_{1}(n)$ in specifying the right - hand side $f$ of the integral equation (8). On the other hand, the operator $P_{1}^{-1}$ is Lipschitz continuous with Lipschitz coefficient equal to $\frac{1}{1-q_{2}}$. Indeed, for any $f, \bar{f} \in X$, we have

$$
\begin{aligned}
& \left\|P_{1}^{-1}(f)-P_{1}^{-1}(\bar{f})\right\|=\left\|x^{(1)}-\bar{x}^{(1)}\right\| \\
& =\left\|x^{(1)}-\bar{x}^{(1)}+A_{2} G_{1}^{-1}\left(x^{(1)}\right)-A_{2} G_{1}^{-1}\left(\bar{x}^{(1)}\right)-\left[A_{2} G_{1}^{-1}\left(x^{(1)}\right)-A_{2} G_{1}^{-1}\left(\bar{x}^{(1)}\right)\right]\right\| \\
& \leq\left\|x^{(1)}-\bar{x}^{(1)}+A_{2} G_{1}^{-1}\left(x^{(1)}\right)-A_{2} G_{1}^{-1}\left(\bar{x}^{(1)}\right)\right\|+\left\|A_{2} G_{1}^{-1}\left(x^{(1)}\right)-A_{2} G_{1}^{-1}\left(\bar{x}^{(1)}\right)\right\| \\
& \leq\left\|P_{1}\left(x^{(1)}\right)-P_{1}\left(\bar{x}^{(1)}\right)\right\|+q_{2}\left\|x^{(1)}-\bar{x}^{(1)}\right\| \\
& =\|f-\bar{f}\|+q_{2}\left\|x^{(1)}-\bar{x}^{(1)}\right\|,
\end{aligned}
$$

so that

$$
\left\|P_{1}^{-1}(f)-P_{1}^{-1}(\bar{f})\right\| \leq \frac{1}{1-q_{2}}\|f-\bar{f}\| .
$$

Hence the substitution of the error $q_{2} \Delta_{1}(n)$ into the right - hand side of the integral equation (8) causes an error of not more than $\frac{q_{2}}{1-q_{2}} \Delta_{1}(n)$ in the corresponding solution $x^{(1)}$. The error of an iteration process in the calculation of $x^{(1)}$ equals $\frac{q_{2}{ }^{n+1}}{1-q_{2}}\|f\|$. Therefore we have

$$
\left\|x_{n}^{(1)}-x^{(1)}\right\| \leq \frac{q_{2}}{1-q_{2}} \Delta_{1}(n)+\frac{q_{2}^{n+1}}{1-q_{2}}\|f\| .
$$

The inverse substitution, i.e., the transition from the variable $x^{(1)}$ to the variable $x$ again introduces the error $\Delta_{1}(n)$. Then the error of approximate solutions $x_{n}$ of Problem 1 gives the estimate 


$$
\begin{aligned}
\left\|x_{n}-x\left(\varepsilon_{0}\right)\right\| & \leq \frac{q_{2}}{1-q_{2}} \Delta_{1}(n)+\Delta_{1}(n)+\frac{q_{2}{ }^{n+1}}{1-q_{2}}\|f\| \\
& =\frac{1}{1-q_{2}} \Delta_{1}(n)+\frac{q_{2}{ }^{n+1}}{1-q_{2}}\|f\| .
\end{aligned}
$$

The approximate solutions of Problem 2 assumes are obtained by iteration processes (18a)-(18c). The values $G_{1}^{-1} G_{2}^{-1}\left(x_{l}^{(2)}\right)$ are calculated by using iteration processes (18a)-(18b). The values $G_{1}^{-1}\left(x_{j}^{(1)}\right)$ are calculated by using the iteration process (18a) with the error

$$
\left\|x_{n}-x^{*}\right\| \leq \frac{q_{1}^{n+1}}{1-q_{1}}\left\|x_{j}^{(1)}\right\| .
$$

We have

$$
\left\|x_{j}^{(1)}\right\| \leq\left\|x_{j}^{(1)}-x_{j-1}^{(1)}\right\|+\cdots+\left\|x_{1}^{(1)}-x_{0}^{(1)}\right\|+\left\|x_{0}^{(1)}\right\| .
$$

Since the operator $G_{2}^{-1}$ is Lipschitz - continuous with Lipschitz coefficient equal to 1 , it follows that

$$
\begin{aligned}
\left\|x_{k}^{(1)}-x_{k-1}^{(1)}\right\| & =\left\|G_{2}^{-1}\left(x_{k}^{(2)}\right)-G_{2}^{-1}\left(x_{k-1}^{(2)}\right)\right\| \\
& \leq\left\|x_{k}^{(2)}-x_{k-1}^{(2)}\right\|, \quad \forall k \in\{1,2, \ldots, n\} .
\end{aligned}
$$

Hence

$$
\left\|x_{j}^{(1)}\right\| \leq\left\|x_{j}^{(2)}-x_{j-1}^{(2)}\right\|+\cdots+\left\|x_{1}^{(2)}-x_{0}^{(2)}\right\|+\left\|x_{0}^{(2)}\right\| .
$$

For any $k \in\{1,2, \ldots, n\}$, we have

$$
\begin{aligned}
\left\|x_{k}^{(2)}-x_{k-1}^{(2)}\right\| & =\left\|F_{1} G_{1}^{-1} G_{2}^{-1}\left(x_{k-1}^{(2)}\right)-F_{1} G_{1}^{-1} G_{2}^{-1}\left(x_{k-2}^{(2)}\right)\right\| \\
& \leq q_{2}\left\|x_{k-1}^{(2)}-x_{k-2}^{(2)}\right\| \leq \cdots \leq q_{2}^{k-1}\left\|x_{1}^{(2)}-x_{0}^{(2)}\right\| .
\end{aligned}
$$

Thus

$$
\begin{aligned}
\left\|x_{j}^{(1)}\right\| & \leq\left(q_{2}{ }^{j-1}+q_{2}{ }^{j-2}+\cdots+q_{2}+1\right)\left\|x_{1}^{(2)}-x_{0}^{(2)}\right\|+\left\|x_{0}^{(2)}\right\| \\
& \leq \frac{1-q_{2}{ }^{j}}{1-q_{2}} x_{1}^{(2)}-x_{0}^{(2)}+\left\|x_{0}^{(2)}\right\| .
\end{aligned}
$$

Since $A_{1}(0)=0$, it follows that

$$
\begin{aligned}
& G_{1}(0)=0+\varepsilon_{0} A_{1}(0)=0 \text { and } \\
& \begin{aligned}
& G_{2}(0)=0+\varepsilon_{0} A_{1} G_{1}^{-1}(0)=0 . \text { Therefore } \\
&\left\|x_{1}^{(2)}-x_{0}^{(2)}\right\|=\left\|A_{2} G_{1}^{-1} G_{2}^{-1}(f)+f-f\right\| \\
&=\left\|A_{2} G_{1}^{-1} G_{2}^{-1}(f)-A_{2} G_{1}^{-1} G_{2}^{-1}(0)\right\| \\
& \leq q_{2}\|f\| .
\end{aligned}
\end{aligned}
$$

Then we have

$$
\begin{aligned}
\left\|x_{j}^{(1)}\right\| & \leq \frac{1-q_{2}^{j}}{1-q_{2}}\left\|x_{1}^{(2)}-x_{\mathrm{O}}^{(2)}\right\|+\left\|x_{\mathrm{O}}^{(2)}\right\| \\
& \leq q_{2} \frac{1-q_{2}^{j}}{1-q_{2}}\|f\|+\|f\| \\
& \leq q_{2} \frac{1-q_{2}^{n}}{1-q_{2}}\|f\|+\|f\| \\
& =\frac{1-q_{2}^{n+1}}{1-q_{2}}\|f\| .
\end{aligned}
$$

Therefore the values $G_{1}^{-1}\left(x_{j}^{(1)}\right)$ are calculated with the error

$$
\left\|x_{n}-x^{*}\right\| \leq \frac{q_{1}{ }^{n+1}}{1-q_{1}} \frac{1-q_{2}{ }^{n+1}}{1-q_{2}}\|f\|=\mu(n) .
$$

Since $\varepsilon_{0} A_{1}$ is a contractive operator with contraction coefficient equal to $q_{1}<1$, the error $\mu(n)$ in specifying the argument of the operator $\varepsilon_{0} A_{1}$ is equivalent to the error $q_{1} \mu(n)$ in specifying the right - hand side $x^{(2)}$ of the equation (12b): $x^{(1)}+\varepsilon_{0} A_{1} G_{1}^{-1}\left(x^{(1)}\right)=x^{(2)}$. Since the operator $G_{2}^{-1}$ is Lipschitz - continuous with Lipschitz coefficient equal to 1 , the substitution of the error $q_{1} \mu(n)$ into the right -hand side of the integral equation (12b) causes an error of not more than $q_{1} \mu(n)$ in the corresponding solution $x^{(1)}$. The error of an iteration process in the calculation of $x^{(1)}$ equals $\frac{q_{1}^{n+1}}{1-q_{1}}\left\|x_{l}^{(2)}\right\|$. For any $l \in\{1,2, \ldots, n\}$, we have

$$
\begin{aligned}
\left\|x_{l}^{(2)}\right\| & \leq\left\|x_{l}^{(2)}-x_{l-1}^{(2)}\right\|+\cdots+\left\|x_{1}^{(2)}-x_{0}^{(2)}\right\|+\left\|x_{0}^{(2)}\right\| \\
& \leq\left(q_{2}^{l-1}+q_{2}^{l-2}+\cdots+q_{2}+1\right)\left\|x_{1}^{(2)}-x_{0}^{(2)}\right\|+\left\|x_{0}^{(2)}\right\| \\
& \leq \frac{1-q_{2}^{l}}{1-q_{2}}\left\|x_{1}^{(2)}-x_{0}^{(2)}\right\|+\left\|x_{0}^{(2)}\right\| \\
& \leq q_{2} \frac{1-q_{2}^{l}}{1-q_{2}}\|f\|+\|f\| \\
& \leq q_{2} \frac{1-q_{2}{ }^{n}}{1-q_{2}}\|f\|+\|f\| \\
& =\frac{1-q_{2}^{n+1}}{1-q_{2}}\|f\| .
\end{aligned}
$$

Then the error of an iteration process in the calculation of $x^{(1)}$ equals $\mu(n)$. Hence

$\delta_{2}(n) \equiv\left\|x_{n}^{(1)}-x^{(1)}\right\| \leq q_{1} \mu(n)+\mu(n)=q_{1} \delta_{1}(n)+\mu(n)$. 
The inverse substitution, i.e., the transition from the variable $x^{(1)}$ to the variable $x$ again introduces the error $\mu(n)$. Consequently, $G_{1}^{-1} G_{2}^{-1}\left(x_{l}^{(2)}\right) \quad$ is calculated with the error

$$
\Delta_{2}(n) \equiv\left\|x_{n}-x^{*}\right\| \leq q_{1} \mu(n)+2 \mu(n)=\delta_{2}(n)+\delta_{1}(n) .
$$

Since $A_{2}$ is a contractive operator with contraction coefficient equal to $q_{2}<1$, the error $\Delta_{2}(n)$ in specifying the argument of the operator $A_{2}$ is equivalent to the error $q_{2} \Delta_{2}(n)$ in specifying the right - hand side $f$ of the equation (13). On the other hand the operator $P_{2}^{-1}$ is Lipschitz continuous with Lipschitz coefficient equal to $\frac{1}{1-q_{2}}$. Indeed, for any $f, \bar{f} \in X$, we have

$$
\begin{aligned}
& \left\|P_{2}^{-1}(f)-P_{2}^{-1}(\bar{f})\right\|=\left\|x^{(2)}-\bar{x}^{(2)}\right\| \\
& =\| x^{(2)}-\bar{x}^{(2)}+A_{2} G_{1}^{-1} G_{2}^{-1}\left(x^{(2)}\right) \\
& \quad-A_{2} G_{1}^{-1} G_{2}^{-1}\left(\bar{x}^{(2)}\right)-\left[A_{2} G_{1}^{-1} G_{2}^{-1}\left(x^{(2)}\right)-A_{2} G_{1}^{-1} G_{2}^{-1}\left(\bar{x}^{(2)}\right)\right] \| \\
& \leq \\
& \quad\left\|x^{(2)}-\bar{x}^{(2)}+A_{2} G_{1}^{-1} G_{2}^{-1}\left(x^{(2)}\right)-A_{2} G_{1}^{-1} G_{2}^{-1}\left(\bar{x}^{(2)}\right)\right\| \\
& \quad+\left\|A_{2} G_{1}^{-1} G_{2}^{-1}\left(x^{(2)}\right)-A_{2} G_{1}^{-1} G_{2}^{-1}\left(\bar{x}^{(2)}\right)\right\| \\
& \leq\left\|P_{2}\left(x^{(2)}\right)-P_{2}\left(\bar{x}^{(2)}\right)\right\|+q_{2}\left\|x^{(2)}-\bar{x}^{(2)}\right\| \\
& =\|f-\bar{f}\|+q_{2}\left\|x^{(2)}-\bar{x}^{(2)}\right\|,
\end{aligned}
$$

so that $\left\|P_{2}^{-1}(f)-P_{2}^{-1}(\bar{f})\right\| \leq \frac{1}{1-q_{2}}\|f-\bar{f}\|$.

Hence the substitution of the error $q_{2} \Delta_{2}(n)$ into the right - hand side of the integral equation (13) causes an error of not more than $\frac{q_{2}}{1-q_{2}} \Delta_{2}(n)$ in the corresponding solution $x^{(2)}$. The error of an iteration process in the calculation of $x^{(2)}$ equals $\frac{q_{2}{ }^{n+1}}{1-q_{2}}\|f\|$. Therefore we have

$$
\left\|x_{n}^{(2)}-x^{(2)}\right\| \leq \frac{q_{2}}{1-q_{2}} \Delta_{2}(n)+\frac{q_{2}{ }^{n+1}}{1-q_{2}}\|f\| .
$$

The inverse substitution, i.e., the transition from the variable $x^{(2)}$ to the variable $x$ again introduces the error $\Delta_{2}(n)$. Then the error of approximate solutions $x_{n}$ of Problem 2 gives the estimate

$$
\begin{aligned}
\left\|x_{n}-x\left(2 \varepsilon_{0}\right)\right\| & \leq \frac{q_{2}}{1-q_{2}} \Delta_{2}(n)+\Delta_{2}(n)+\frac{q_{2}{ }^{n+1}}{1-q_{2}}\|f\| \\
& =\frac{1}{1-q_{2}} \Delta_{2}(n)+\frac{q_{2}{ }^{n+1}}{1-q_{2}}\|f\| .
\end{aligned}
$$

By using similar arguments for the problem $k$ : $x+k \varepsilon_{0} A_{1}(x)+A_{2}(x)=f, k \in[1, N]$, we obtain the estimation

$\left\|x_{n}-x\left(k \varepsilon_{0}\right)\right\| \leq \frac{1}{1-q_{2}} \Delta_{k}(n)+\frac{q_{2}{ }^{n+1}}{1-q_{2}}\|f\|$,

where

$\Delta_{k}(n) \leq \delta_{k}(n)+\delta_{k-1}(n)+\cdots+\delta_{1}(n)$,

and

$$
\delta_{h}(n) \leq q_{1}\left[\delta_{h-1}(n)+\cdots+\delta_{1}(n)\right]+\mu(n), 1 \leq h \leq k .
$$

We shall rewrite inequality (25) in the following form

$$
\delta_{k}(n) \leq \mu(n)+q_{1} \sum_{h=1}^{k-1} \delta_{h}(n), \delta_{1}(n) \leq \mu(n), k=2,3, \ldots, N .
$$

By virtue of the discrete analogue of the well known Bellman-Gronwall lemma (see [4, Theorem 1.28]), from inequality (26) we get

$$
\begin{array}{r}
\delta_{k}(n) \leq \mu(n) \prod_{h=1}^{k-1}\left(1+q_{1}\right) \leq \mu(n) \prod_{h=1}^{k-1} e^{q_{1}}=\mu(n) e^{q_{1}(k-1)}, \\
k=1,2, \ldots, N .
\end{array}
$$

Hence the inequality (24) can be written as

$$
\Delta_{k}(n) \leq \sum_{h=1}^{k} \delta_{h}(n) \leq \mu(n) \sum_{h=1}^{k} e^{q_{1}(h-1)}=\mu(n) \frac{e^{k q_{1}}-1}{e^{q_{1}}-1} .
$$

Consequently, we can rewrite the estimation of the error (23) for problem $k$ as the form

$$
\left\|x_{n}-x\left(k \varepsilon_{0}\right)\right\| \leq \frac{1}{1-q_{2}} \mu(n) \frac{e^{k q_{1}}-1}{e^{q_{1}}-1}+\frac{q_{2}^{n+1}}{1-q_{2}}\|f\| .
$$

Substituting $N$ for $k$ and by (22), we obtain (21). This completes the proof of the theorem.

Remark 2.1. These results are extension of the known result on the application of the method of contractive mapping and PCM for solving operator equation of the second kind. Indeed, we consider the two following special cases. When 
$A_{1} \equiv 0$, the equation (6) has form $x+A_{2}(x)=f$, where $A_{2}$ is a contractive operator. When $A_{2} \equiv 0$, the equation (6) has form $x+A_{1}(x)=f$, where $A_{1}$ is monotone and Lipschitz - continuous.

Example. Consider the following nonlinear Fredholm integral equation $x(t)+\int_{0}^{1}\left[\frac{9}{2} t s x(s)+\frac{2}{3} t \cos (x(s))\right] d s$ $=\sqrt{t}+\frac{7+20 \sin (1)+20 \cos (1)}{15} t, 0 \leq t \leq 1$.

We define two operators $A_{1}, A_{2}: L^{2}[a ; b] \rightarrow L^{2}[a ; b]$ by

$$
\begin{aligned}
& \left(A_{1} x\right)(t)=\frac{9}{2} \int_{0}^{1} t s x(s) d s \\
& \left(A_{2} x\right)(t)=\frac{2}{3} \int_{0}^{1} t \cos (x(s)) d s, \forall x \in L^{2}[a ; b] .
\end{aligned}
$$

It is easy to verify that $A_{1}$ is monotone and Lipschitz - continuous with Lipschitz coefficient $L=\frac{3}{2}, A_{2}$ is a contractive operator with contraction coefficient $\quad q_{2}=\frac{2 \sqrt{3}}{9}<1 . \quad$ Consequently, all conditions of

Theorem 2.1 are satisfied. Then the equation (27) has a unique solution. By applying the iteration processes (19a)-(19d) with $N=2, n=30,50$ we obtain approximate solutions of this equations as follows

\begin{tabular}{|c|l|l|}
\hline$n$ & Approximate solution & Error \\
\hline 30 & $0.124238398349 \times 10^{-3} t+\sqrt{t}$ & $0.8963691179 \times 10^{-2}$ \\
\hline 50 & $0.635113898 \times 10^{-6} t+\sqrt{t}$ & $0.2842576447 \times 10^{-4}$ \\
\hline
\end{tabular}

Table. Approximate solutions and corresponding errors of the equation (27).

\section{CONCLUSION}

In this paper, we have presented a new extension of the PCM for solving operator equation of the second kind. The reason for the powerful extension is that the new decomposition strategy for nonlinear operator, which increase the applicability of the method. The obtained results have been illustrated by an example.

\section{REFERENCES}

[1] Bernstein, S. N. (1906). Sur la généralisation du problème de Dirichlet, Math. Ann. 62:253-271.

[2] Gaponenko, Y. L. (1986). The parameter - extension method for an equation of the second kind with a Lipschitz - continuous and monotonic operator. Comput. Maths. Math. Phys, 26:11231131.

[3] Leray, J., Schauder, J. (1934). Topologi et équations fonctionnelles. Ann. Sci. Éc. Norm. Supér. 51:45-78.

[4] Phat, V.N. (2001). Introduction to mathematical control theory. Vietnam National University Press, Hanoi, Vietnam.

[5] Trenogin, V. A. (1990). Functional Analysis. Nauka, Moscow.

[6] Trenogin, V. A. (1996). Global invertibility of nonlinear operator and the method of continuation with respect to a parameter, Dokl. Akad. Nauk, 350:1-3.

[7] Trenogin, V. A. (1996). Locally invertible operator and parameter continuation method, Funktsional. Anal. i Prilozhen, 30:93-95. 